\title{
Control of growth orientation in as-deposited epitaxial iron-rich nickel ferrite spinel
}

\author{
Jon E. Bratvold, Henrik H. Sønsteby, ${ }^{\text {a) }}$ Ola Nilsen, and Helmer Fjellvåg \\ Department of Chemistry, University of Oslo, Sem Scelands vei 26, 0371 Oslo, Norway
}

(Received 19 November 2018; accepted 19 December 2018; published 9 January 2019)

\begin{abstract}
The authors here report epitaxial growth of the ferrimagnet $\mathrm{Ni}_{x} \mathrm{Fe}_{3-x} \mathrm{O}_{4}$ (NFO) by atomic layer deposition at low temperatures. Films grow epitaxially at a reactor temperature of $250{ }^{\circ} \mathrm{C}$ and require no further postannealing treatment. (100)-, (110)-, and (111)-oriented thin films of NFO are attainable by using $\mathrm{MgO}(100) / \mathrm{LaAlO}_{3}(100), \mathrm{LaAlO}_{3}$ (110), and $\mathrm{Al}_{2} \mathrm{O}_{3}(001)$ substrates, respectively. This means that the direction of the easy axis of magnetization relative to the film surface can be selected by the choice of substrate. The authors carefully study nucleation onset and growth dynamics by varying the number of cycles used to deposit the films. This shows that the initial growth is mostly inhibited by the Fe-species, creating a small composition gradient for the first few nanometers of the film. The growth rate varies as a result of the choice of substrate, explained by the direction of the close packed layers in the film. The authors believe that the ability to deposit epitaxial ferrimagnetic films with angstrom thickness control at low temperatures will make a major impact on the possibility to grow multiferroic stacks and on low temperature monolithic device integration where a spontaneously magnetized sample is required. Published by the AVS.

https://doi.org/10.1116/1.5082012
\end{abstract}

\section{INTRODUCTION}

Thin films with spontaneous magnetization are essential in a range of modern applications, including spintronics, magnetic data storage, magneto-optics, and in novel composite multiferroics. ${ }^{1-7}$ A prerequisite for the latter is epitaxial integration with a ferroelectric or ferroelastic compound, in addition to an applicable magnetostriction: changes in material dimensions upon perturbation by a magnetic field. Although ferromagnetic metals (such as Terfenol D) offer the highest known magnetostriction, monolithic integration in functional devices is often a challenge due to high processing temperatures. ${ }^{8}$ Lattice mismatch toward other components in the composites creates detrimental strain, resulting in polycrystallinity and pinning with accompanying complex magnetic behavior. As a result, there has been a surge of interest in ferro- or ferrimagnetic complex oxides with perovskite- or spinel-type structures. These can often be integrated in heteroepitaxial stacks, have flexible stoichiometry for tuning functional parameters, and can withstand harsh environments. ${ }^{9}$

One such material type is the nickel ferrite inverse spinel (Fd3m, SG\# 227), $\mathrm{Ni}_{x} \mathrm{Fe}_{3-x} \mathrm{O}_{4}$ (NFO). In this structure, half of the $\mathrm{Fe}^{3+}$ cations occupy the tetrahedral A sites, while the remaining $\mathrm{Fe}^{3+} / \mathrm{Fe}^{2+}$ and $\mathrm{Ni}^{2+}$ cations are distributed on the octahedral B sites. The magnetic structure of NFO is explained by the traditional Néel-model of ferrimagnetism. A strong superexchange interaction between nearest neighbor A and B sites via oxygen results in antiparallel alignment of the local magnetic moments. ${ }^{10}$ This coupling results in the cancellation of magnetic moments of the $\mathrm{Fe}^{3+}$ cations on tetrahedral

Note: This paper is part of the Special Topic Collection on Complex Oxides.

${ }^{a)}$ Electronic mail: henrik.sonsteby@kjemi.uio.no sites and half of the octahedral sites, and a net magnetic moment setup from the $\mathrm{d}^{8} \mathrm{Fe}^{2+} / \mathrm{Ni}^{2+}$ cations on the rest of the octahedral sites (Fig. 1).

Bulk, stoichiometric NFO $\left(\mathrm{NiFe}_{2} \mathrm{O}_{4}\right)$ has a high Curie temperature $\left(T_{c}=\sim 500{ }^{\circ} \mathrm{C}\right)$, a theoretical room temperature saturation magnetization of $\sim 270 \mathrm{emu} / \mathrm{cm}^{3}$, is negatively magnetostrictive $\left(\lambda_{\mathrm{s}}=-26 \cdot 10^{-6}\right)$, and exhibits a strong magnetic anisotropy. ${ }^{11}$ This unique combination of properties makes it a viable material for use in magnetoelectric multilayer stacks. ${ }^{12-14}$ In addition, all these functional parameters can be varied within the stoichiometric range of the solid solution, with $0<x<1\left(\mathrm{Fe}_{3} \mathrm{O}_{4} \rightarrow \mathrm{NiFe}_{2} \mathrm{O}_{4}\right)$.

NFO thin films have been synthesized by a range of deposition techniques, including pulsed laser deposition, molecular beam epitaxy, chemical vapor deposition, rf sputtering, sol-gel techniques, and more recently atomic layer deposition (ALD). ${ }^{15-21}$ A common trait for these reports is high processing temperatures and/or high postannealing temperatures that make monolithic device integration difficult. The exception is ALD-grown films reported by Chong et al. in 2010, which were polycrystalline as deposited on silicon at a reaction temperature of just $200{ }^{\circ} \mathrm{C} .{ }^{21}$

ALD is a layer-by-layer self-limiting deposition technique, utilizing surface saturation of precursor molecules that are pulsed sequentially into a reaction chamber. ${ }^{22}$ This facilitates the growth of thin films with thickness control on the submonolayer level, extreme conformality on high aspect ratio substrates, and excellent stoichiometric control. Deposition of multilayer systems is attainable by expanding the amount of precursors that can be sequentially pulsed into the reaction chamber. ALD also enables wafer-scale batch deposition of conformal films, holding it in high regard for industrial upscaling. The technique has traditionally been used by the 


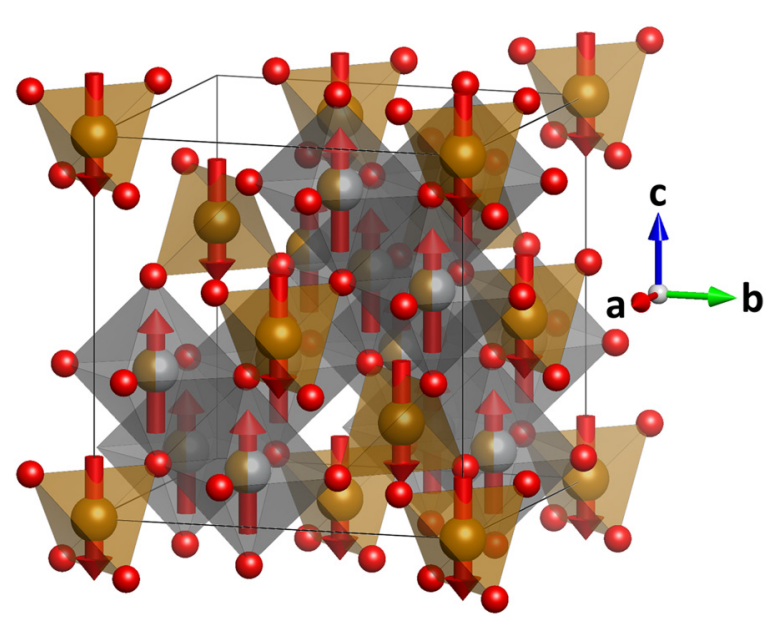

FIG. 1. Structure with accompanying atomic magnetic moments of $\mathrm{Ni}_{x} \mathrm{Fe}_{3-x} \mathrm{O}_{4}$ Bronze (dark) colored spheres in tetrahedral coordination are iron, whereas mixed bronze (dark) and silver (light) colored spheres in octahedral coordinatioin are nickel or iron.

industry to deposit pinhole free high- $\kappa$ materials, but over the past decade, interest in depositing epitaxial complex oxides by ALD has rapidly increased. ${ }^{23}$

Chong et al. obtained polycrystalline films with a spontaneous magnetization at room temperature using commercial homoleptic cyclopentadienyl complexes of $\mathrm{Ni}^{2+}$ and $\mathrm{Fe}^{2+}$ [nickelocene $\mathrm{Ni}(\mathrm{cp})_{2}$ and ferrocene $\left.\mathrm{Fe}(\mathrm{cp})_{2}\right]^{21}{ }^{21}$ The relatively small size of these precursor molecules gives rise to a chemisorbed areal density that facilitates crystal growth, which is uncommon for ALD complex oxides. This means that no postannealing is needed to achieve the ferrimagnetic structure. However, due to the large magnetic anisotropy of NFO, it is important to control crystal orientation to have the easy axis of magnetization point in a specific direction. The desired orientation varies in accordance with application, as you can effectively control the magnetic hardness of the material by selectively tuning the direction of the easy axis.

In this work, we report the control of the crystal orientation in $\mathrm{Ni}_{x} \mathrm{Fe}_{3-x} \mathrm{O}_{4}$ thin films by careful selection of substrates. We deposit a selection of stoichiometries in the $\mathrm{Ni}_{x} \mathrm{Fe}_{3-x} \mathrm{O}_{4}$ family for the proof of chemical control and focus on a specific member $\left(\mathrm{Ni}_{0.6} \mathrm{Fe}_{2.4} \mathrm{O}_{4}\right)$ for the control of growth orientation. This composition is emphasized due to its possible use in multiferroic stacks, where its combination of properties is especially well suited. ${ }^{24}$ We finally discuss variation in growth dynamics as a result of the orientation of the film. We believe that the possibility to deposit highly oriented thin films with a direction-controlled uniaxial spontaneous magnetization at low temperatures opens for expanding the toolbox of novel composite multiferroic functionality.

\section{EXPERIMENT}

Thin films were deposited in an F-120 Sat reactor (ASM Microchemistry). All films were deposited at a reactor temperature of $250^{\circ} \mathrm{C}$. Nitrogen purging gas was supplied from a Nitrox UHPN 3001 generator producing 99.9995\% $\left(\mathrm{N}_{2}+\mathrm{Ar}\right)$ and maintained at a $300 \mathrm{~cm}^{3} \mathrm{~min}^{-1}$ primary flow rate. Reactor operating pressure was maintained at 2.8 mbar throughout the depositions.

All depositions were carried out using $\mathrm{Ni}(\mathrm{acac})_{2}(\mathrm{acac}=$ acetylacetonate, Sigma Aldrich, 95\%) and $\mathrm{Fe}(\mathrm{cp})_{2}(\mathrm{cp}=$ cyclopentadienyl, Sigma Aldrich, 98\%) as metal precursors. Both precursors were resublimated in order to increase purity. $\mathrm{O}_{3}$ was used as the oxygen source, made from $\mathrm{O}_{2}$ gas (AGA, 99.999\%) using an OT-020 ozone generator (Ozone Technology) with a claimed concentration of 15 mass $\% \mathrm{O}_{3}$ in $\mathrm{O}_{2}$. Pulse durations were 2, 2, and $3 \mathrm{~s}$ for $\mathrm{Ni}(\mathrm{acac})_{2}, \mathrm{Fe}(\mathrm{cp})_{2}$, and $\mathrm{O}_{3}$, respectively. Purge durations were in the 1.5-3 s range depending on the preceding precursor pulse. These pulse and purge times are based on experience with achieving self-limiting growth with the same precursors for deposition of similar materials in the same ALD-reactors throughout our prior studies.

The films were deposited on $1 \times 1 \mathrm{~cm}^{2} \mathrm{Si}(100)$ substrates for the characterization of thickness and conformality, while $3 \times 3 \mathrm{~cm}^{2} \mathrm{Si}$ (100) substrates were used for compositional analysis. Selected compositions were also deposited on $\mathrm{LaAlO}_{3}$ (100), $\mathrm{LaAlO}_{3}$ (110), $\mathrm{MgO}$ (100), and $\mathrm{Al}_{2} \mathrm{O}_{3}$ (001) single crystal substrates for the facilitation of epitaxial growth and the control of growth direction.

Thin film thickness was measured using a J. A. Woollam alpha-SE spectroscopic ellipsometer in the range of 390$900 \mathrm{~nm}$. A Cauchy-function was successfully used to model the collected data. Complementary thickness measurements were carried out using x-ray reflectivity (XRR), on a PANalytical Empyrean diffractometer equipped with a $\mathrm{Cu} K_{\alpha}(\lambda=1.5406 \AA)$ source and a parallel beam mirror, powered at $45 \mathrm{kV}$ and $40 \mathrm{~mA}$.

$\mathrm{X}$-ray diffraction measurements were performed on a Bruker AXS D8 Discover diffractometer equipped with a LynxEye strip detector and a Ge (111) focusing monochromator, providing $\mathrm{Cu} K_{\alpha 1}$ radiation.

Reciprocal space maps for identification of epitaxy were collected at the European Synchrotron Radiation Facility in Grenoble, France. The experiment was carried out in a surface $\mathrm{X}$-ray diffraction setup at the ID03 beamline, with a monochromatic beam of energy $20 \mathrm{keV}$.

Chemical composition was analyzed using a Panalytical Axios Max Minerals x-ray fluorescence (XRF) system equipped with a $4 \mathrm{~kW}$ Rh-tube. The system is running with Omnian and Stratos options for standardless measurements of thin films.

Morphological studies were performed using a Park Systems XE-70 AFM equipped with a standard NHCR tip. Data were analyzed using the GWYDDION 2.43 SPM visualization software. ${ }^{25}$

\section{RESULTS AND DISCUSSION}

A common approach for depositing ternary oxides by ALD is combining binary oxide processes at a ratio resulting in the wanted stoichiometry in the film. As the growth rates of the different processes are not necessarily equal, this typically results in a pulsed ratio that that does not map linearly with the deposited cation stoichiometry. We used this approach in the work presented here, studying the relationship in the pulsed to deposited ratio in the iron-rich pulsing regime 


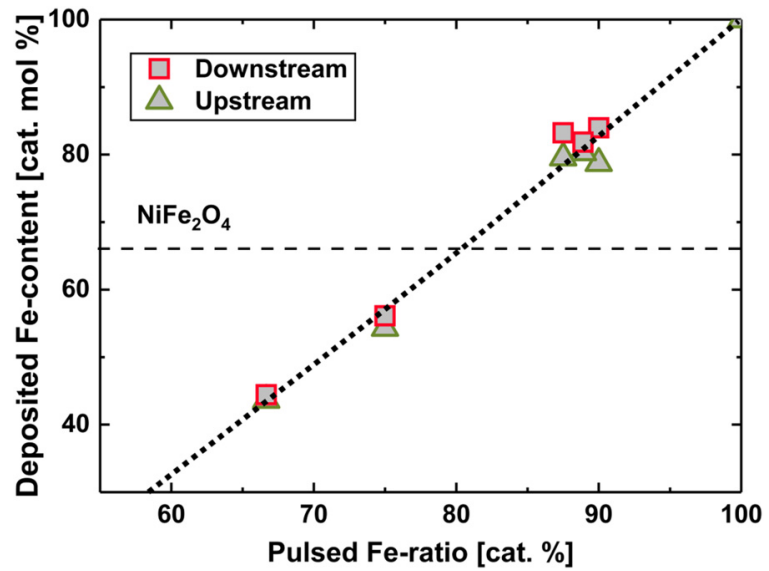

FIG. 2. Deposited Fe-content in cation $\%$ as a function of the ratio between pulsed $\mathrm{Fe}(\mathrm{cp})_{2}$ and $\mathrm{Ni}(\mathrm{acac})_{2}$ as measured by standardless XRF. Green triangles are concentrations close to the precursor outlet, while red squares are close to the exhaust. The black dotted line is a guide-of-eye showing a slightly banana-shaped relationship between the pulsed and deposited cation concentrations. The horizontal black dashed line indicates the stoichiometric $\mathrm{NiFe}_{2} \mathrm{O}_{4}$ composition.

(pulsed Fe $>60$ cation \%). From Fig. 2, it is clear that the $\mathrm{Fe}_{x} \mathrm{O}$-part of the process exhibits slower growth than the $\mathrm{Ni}_{x} \mathrm{O}$-part, manifested in a nonlinear relationship between the pulsed and deposited cation ratios. This is expected from previously reported growth rates of the $\mathrm{Fe}(\mathrm{cp})_{2}+\mathrm{O}_{3}$ and $\mathrm{Ni}(\mathrm{acac})_{2}+\mathrm{O}_{3}$ processes. $^{26,27}$

It is also clear from Fig. 2 that the stoichiometry of the $\mathrm{Ni}_{x} \mathrm{Fe}_{3-x} \mathrm{O}_{4}$ system can be controlled for a large range of $x$ with a close to linear relationship in the Fe-rich region. Note that Fe-concentrations close to $100 \%$ will not result in magnetite $\left(\mathrm{Fe}_{3} \mathrm{O}_{4}\right)$, but in hematite $\left(\mathrm{Fe}_{2} \mathrm{O}_{3}\right) .{ }^{26}$ The small difference in upstream (close to the precursor inlet) and downstream (close to the exhaust) cation concentrations indicate that the chemical uniformity of the process is high.

Our goal was to study growth dynamics and orientation control in iron-rich nickel ferrite $\left(\mathrm{Ni}_{x} \mathrm{Fe}_{3-x} \mathrm{O}_{4}, 0.4<x<0.8\right)$. We found that 88 cation $\%$ pulses of $\mathrm{Fe}(\mathrm{cp})_{2}$ results in a deposited composition of $\mathrm{Ni}_{0.6} \mathrm{Fe}_{2.4} \mathrm{O}_{4}$, believed to be optimal

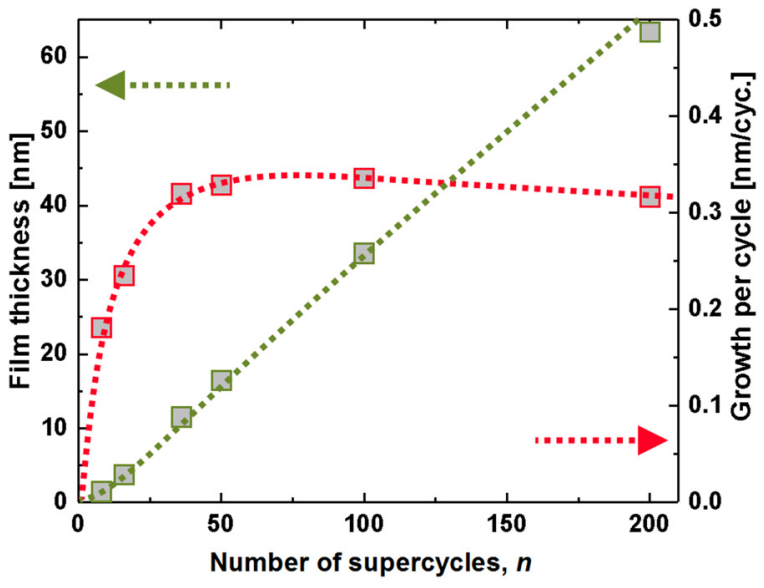

FIG. 3. Film thickness (green) and growth per supercycle (red) as a function of the number of employed supercycles. The green dotted line is a guide-of-eye line showing the estimated relationship between the pulsed number of supercycles and the film thickness. The red dotted line is a guide-of-eye line indicating the relationship between the growth per supercycle and the employed number of supercycles.

for the use in magnetoelectric stacks. This was obtained using the following pulsing sequence:

$$
n \times\left\{8 \times\left[\mathrm{Fe}(\mathrm{cp})_{2}+\mathrm{O}_{3}\right]+\left[\mathrm{Ni}(\mathrm{acac})_{2}+\mathrm{O}_{3}\right]\right\} .
$$

The thickness of the film here is only dependent of the number of supercycles, $n$. Time parameters were used as described in Sec. II. We studied the relationship between $n$ and the deposited thickness by keeping the pulsed ratio constant and only varying the number of supercycles (Fig. 3).

The growth is clearly inhibited for the first 20-30 supercycles, after which the growth per cycle stabilizes and the thickness increases almost linearly. This inhibition is thought to originate from slow nucleation on the pristine substrate surface and is commonly seen for as-deposited crystalline thin films. ${ }^{28-30}$ An interesting question is whether the stoichiometry is preserved throughout the nucleation stage or if one of the precursors dominate the growth inhibition.

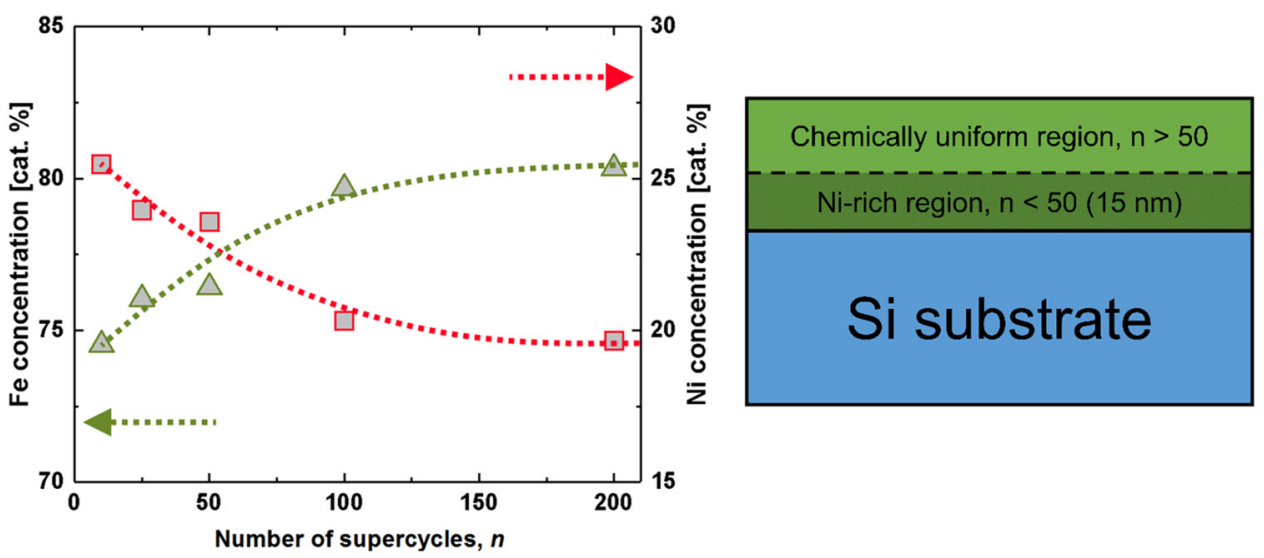

FIG. 4. Left: Iron (green) and nickel (red) cation concentration determined by standardless XRF as a function of the employed number of supercycles, n. The red and green dotted lines are guide-of-eye lines indicating the relationship between the employed number of supercycles and the cation content. Right: A sketch of the sample indicating a nickel-rich interfacial region. 


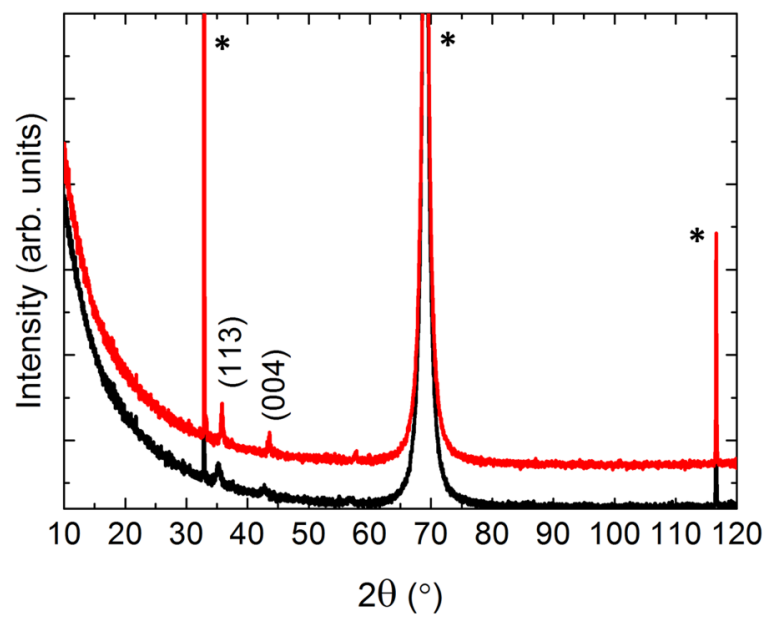

FIG. 5. Specular x-ray diffractogram $(\theta-2 \theta)$ for as-deposited (black) and annealed (red) nickel ferrite on untreated silicon substrates. Black asterisks $(*)$ indicate substrate reflections. The annealed sample is offset in y-scale for easy comparison.

To examine this, we studied the film composition as a function of the number of supercycles (Fig. 4).

It is evident that the Fe:Ni ratio increases with the first 100 cycles, before it reaches a stable value at approximately the desired $\mathrm{Ni}_{0.6} \mathrm{Fe}_{2.4} \mathrm{O}_{4}$ composition. It is worthwhile noting that the variation is relatively small, with the composition being close to $\mathrm{Ni}_{0.7} \mathrm{Fe}_{2.3} \mathrm{O}_{4}$ for less than 50 supercycles. Also note that the sensitivity of the XRF-instrument is lower for very thin films $(<10 \mathrm{~nm})$, which might interfere with the data. The trend in the composition variation does indicate, however, that there is stronger growth inhibition for $\mathrm{Fe}_{x} \mathrm{O}$ in the initial cycles.

We proceeded by studying the crystallinity of asdeposited and postannealed $\left(650^{\circ} \mathrm{C}\right.$ for $\left.15 \mathrm{~min}\right)$ films on silicon substrates (Fig. 5). There are traces of polycrystalline unoriented NFO in both cases, with slightly sharper reflections for the annealed case (red line). This is in agreement with the previously published results by Chong et $_{\text {al. }}{ }^{21}$

To try to obtain the control of growth orientation (i.e., easy axis of magnetization), we deposited the films on a range of single crystal oxide substrates thought to facilitate the epitaxial growth. $\mathrm{MgO}$ has a very close lattice match with $\mathrm{NFO}\left(a_{\mathrm{NFO}} / 2 \approx a_{\mathrm{MgO}}\right)$ and should result in cube-oncube (100)\|(100)-growth. Pseudocubic $\mathrm{LaAlO}_{3}$ has a somewhat smaller cell, but is commonly used to obtained strained cube-on-cube (100)||(100)-growth of spinel ferrites as well. Furthermore, we employed $\mathrm{LaAlO}_{3}$ (110) substrates in an attempt to deposit (110)-oriented NFO films. Finally, $\mathrm{Al}_{2} \mathrm{O}_{3}$ (001) ( $c$ cut) substrates have a close lattice match with the cube diagonal of NFO $\left(\sqrt{3} a_{\mathrm{NFO}} \approx 3 \quad a_{\mathrm{Al2O} 3}\right)$ and were utilized to try to obtain (111)-oriented NFO films. Expected in-plane strain from lattice mismatch for different substrates is summarized in Table I.

Specular XRD was recorded for $\mathrm{Ni}_{0.6} \mathrm{Fe}_{2.4} \mathrm{O}_{4}$ films $(n=200)$, both as-deposited and postannealed at $6500^{\circ} \mathrm{C}$ for $15 \mathrm{~min}$, on all the employed substrates (Fig. 6). All as-deposited films are crystalline and exhibit a single out-of-plane orientation in agreement

TABLE I. Surface lattice parameters and theoretical lattice mismatches in the NFOllsubstrate-systems. The lattice mismatch is calculated as $\varepsilon=\left(d_{\mathrm{NFO}}-d_{\mathrm{sub}}\right) /$ $d_{\mathrm{NFO}}$, where $\varepsilon$ is the mismatch and $d$ indicates lattice parameters of the explored systems.

\begin{tabular}{lcccc}
\hline \hline & $\begin{array}{c}\text { Surface } \\
\text { lattice } \\
\text { parameter, } a \\
(\AA)\end{array}$ & $\begin{array}{c}\text { Surface } \\
\text { lattice } \\
\text { parameter, } b \\
(\AA)\end{array}$ & $\begin{array}{c}\text { Lattice } \\
\text { mismatch } \\
\text { in } a \\
(\%)\end{array}$ & $\begin{array}{c}\text { Lattice } \\
\text { mismatch } \\
\text { in } b \\
(\%)\end{array}$ \\
\hline $\mathrm{MgO}(100)$ & 4.22 & 4.22 & +1.2 & +1.2 \\
$\mathrm{LaAlO}_{3}(100)$ & 3.81 & 3.81 & -8.6 & -8.6 \\
$\mathrm{LaAlO}_{3}(110)$ & 3.81 & 2.69 & -8.6 & -8.8 \\
$\mathrm{Al}_{2} \mathrm{O}_{3}(001)$ & 4.76 & 4.76 & -1.3 & -1.3 \\
\hline \hline
\end{tabular}

with the theorized film-to-substrate relationships. Postannealing does not affect orientation, but lowers the FWHM of the reflections due to the growth of larger crystallites. The only exemption is NFO (100)\|MgO (100), in which the reflections are broader in the annealed sample. This may indicate that there is a longer relaxation distance in the annealed samples. Note that the variation is small and that the films are close to unstrained in both cases.

We investigated the origin of broadening by WilliamsonHall analysis (see supplementary material for WilliamsonHall plots). ${ }^{33}$ The values for the $y$-intercepts in all the Williamson-Hall plots are very small, indicating that crystallites are traversing all the way from the interface to the thin film surface. Furthermore, as expected, there is a significant relaxation (i.e., reduced strain) upon annealing. NFO on $\mathrm{MgO}$ (100) is nearly unstrained, which is in agreement with the close lattice match. A significant strain is observed for NFO on $\mathrm{LaAlO}_{3}$ (100), due to the relatively large lattice mismatch.

NFO||MgO(100)

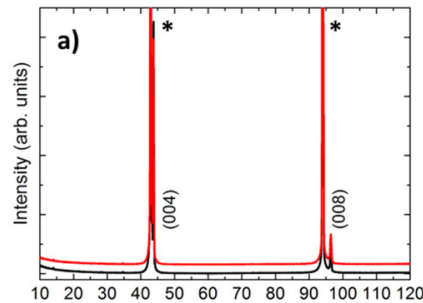

$2 \theta\left(^{\circ}\right)$

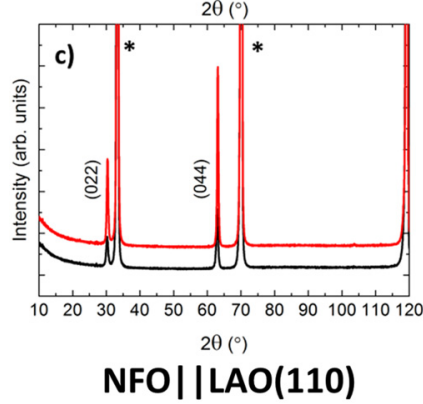

FIG. 6. Specular x-ray diffractogram $(\theta-2 \theta)$ for as-deposited (black) and annealed (red) nickel ferrite on $\mathrm{MgO}$ (100) (top left), $\mathrm{LaAlO}_{3}$ (100) (top right), $\mathrm{LaAlO}_{3}$ (bottom left), and $\mathrm{Al}_{2} \mathrm{O}_{3}$ (bottom right). Black asterisks (*) indicate substrate reflections. The annealed sample is offset in y-scale for easy comparison. 


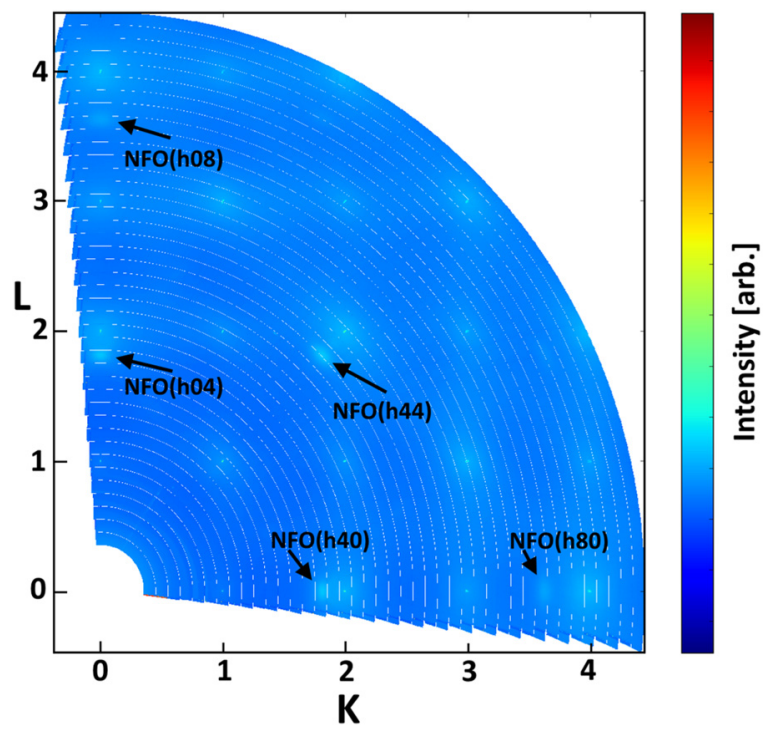

FIG. 7. Reciprocal space map for $\mathrm{NFO} \mathrm{LaAlO}_{3}(100)$ films showing a slice in reciprocal space with $\mathrm{h} \approx 1$. The $x$ and $y$ axes indicate $\mathrm{K}$ and $\mathrm{L}$ values in $\mathrm{LaAlO}_{3}$ coordinates. Unmarked reflections are substrate reflections. White circular lines are an artifact of data reduction.

NFO on $\mathrm{LaAlO}_{3}$ (110) is strained as deposited, but is almost fully relaxed after annealing. For $\mathrm{NFO}$ on $\mathrm{Al}_{2} \mathrm{O}_{3}(001)$, there are two sets of linear curves in the Williamson-Hall plots. Reflections with even indices are considerably less broad when compared to their odd counterparts. The close packed layers in the NFO-structure are in the (111)-direction. Some layers contain only $\mathrm{Fe}^{3+}$, whereas other layers contain a mix of $\mathrm{Ni}^{2+}$ and $\mathrm{Fe}^{3+} / \mathrm{Fe}^{2+}$. If there is a composition distribution along the normal of the film, this will manifest itself in the odd $(h h h)$-reflections, as only reflections with odd indices probe the mixed composition layers. This is in agreement with our hypothesis from Fig. 3, which indicates a Ni-rich phase close to the interface between substrate and film.

A single orientation is not a guarantee for epitaxy, although as-deposited orientation is commonly accompanied by an epitaxial relationship. We chose to examine the sample with the highest theoretical lattice mismatch as a proof-of-concept that the films do indeed grow epitaxially. Surface x-ray diffraction at ID03@ESRF was used to evaluate the in-plane orientation for $\mathrm{NFOllaAlO}_{3}$. A full reciprocal space map (RSM) is shown in Fig. 7, with accompanying single-reflection RSMs in Fig. 8.

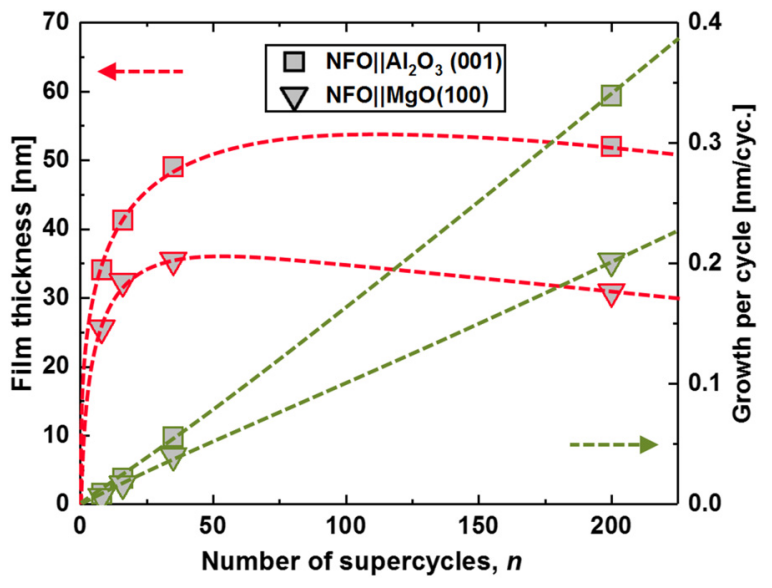

FIG. 9. Film thickness (red, left axis) and growth per supercycle (green, right axis) for $\mathrm{NFO}$ on $\mathrm{Al}_{2} \mathrm{O}_{3}$ (001) (squares) and $\mathrm{MgO}$ (100) (triangles), as a function of the number of supercycles, $n$. The green (right axis) dashed line is a guide-of-eye line showing the estimated relationship between the pulsed number of supercycles and the film thickness. The red (right axis) line is a guide-of-eye line indicating the relationship between the growth per cycle and the employed number of cycles.

The RSMs were collected for $h \approx 1$ (in $\mathrm{LaAlO}_{3}$-space) as part of scanning crystal truncation rods, so in principle, it is the $(2 \mathrm{kl})$ reflections for NFO that is visible. The broadening in the reflections is small, and there are no signs of polycrystallinity. This confirms an epitaxial relationship of NFO(100)| $\mathrm{NFO}[001] \| \mathrm{LaAlO}_{3}(100) \mid \mathrm{LaAlO}_{3}[001]$. Further analysis of the diffuse scattering and intensity distribution in the RSMs is related to the surface- and interface structure of the samples. This is not within the scope of this work and will be presented in a separate paper.

To strengthen the theory of epitaxy, we studied the growth on different substrates by measuring the thickness of the films with a varying number of cycles by XRR (Fig. 9). If the films indeed crystallize upon deposition, there should be a large variation in their thicknesses on the different substrates. (100)-oriented films growing cube-on-cube will grow slowly as the cube face facets are off-angle with the close packed layers. On the other hand, (111)-oriented films should grow faster as the (111)-planes coincide with the close packed layers. This should manifest in higher growth per cycle for the (111)-oriented films. Using the formula proposed by Ylilammi et al., we calculated the theoretical growth rate in (100)and (111)-directions to be $0.23 \AA /$ cycle and $0.34 \AA /$ cycle,
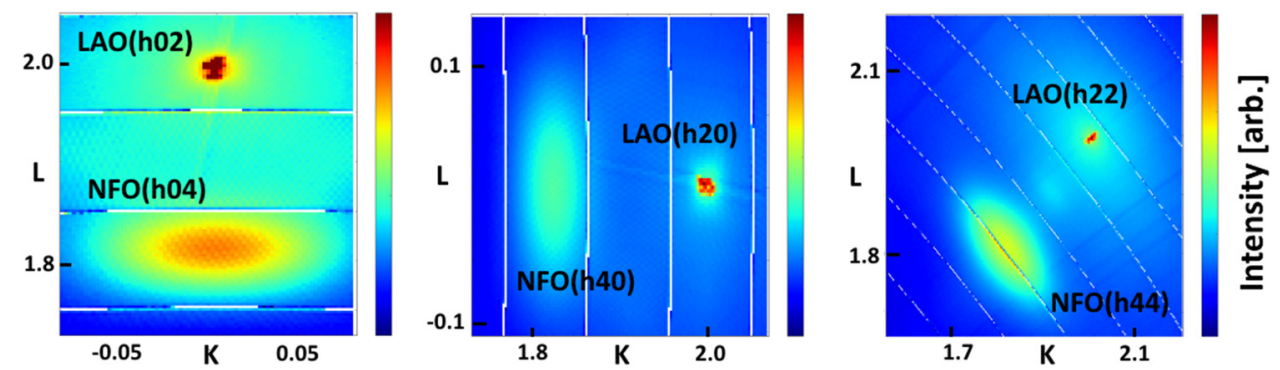

FIG. 8. High-resolution reciprocal space maps of the NFO (204)- (left), NFO (240)- (middle), and NFO (444)- (right) reflections. The $x$ and $y$ axes indicate $\mathrm{K}$ and $\mathrm{L}$ values in $\mathrm{LaAlO}_{3}$ coordinates. White lines are an artifact of data reduction. 


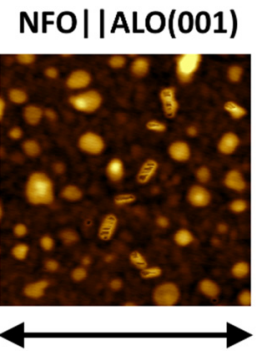

$500 \mathrm{~nm}$

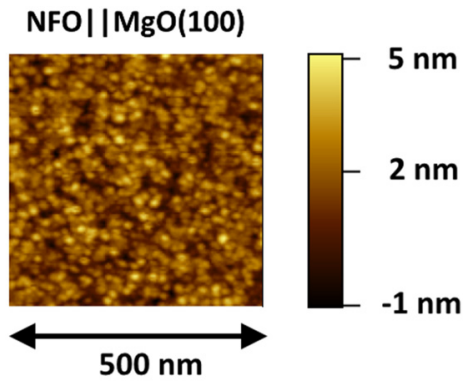

FIG. 10. Surface morphology of $\mathrm{Ni}_{0.6} \mathrm{Fe}_{2.4} \mathrm{O}_{4}$ thin films on $\mathrm{Al}_{2} \mathrm{O}_{3}$ (001) (left) and $\mathrm{MgO}(100)$ (right), as collected by atomic force microscopy in tapping mode. Images are scaled to the same z-range for easy comparison.

respectively, if the films grow epitaxially as deposited. ${ }^{31}$ (See supplementary material for calculations. $)^{33}$

A clear difference between the growth per cycle on the two substrates is observed (Fig. 9). NFO (100) on $\mathrm{MgO}$ (100) grows slower than NFO (111) on $\mathrm{Al}_{2} \mathrm{O}_{3}$ (001), with experimental growth rates in the linear region of $0.21 \AA /$ cycle and $0.31 /$ cycle, respectively. This is in remarkably good agreement with the theoretical growth rates for epitaxial growth.

Finally, we studied the surface morphology of NFO on $\mathrm{Al}_{2} \mathrm{O}_{3}$ (001) and $\mathrm{MgO}$ (100), Fig. 10. If the films do indeed grow epitaxially, there should be a large difference between the morphology on the two different substrates. ${ }^{32}$ (100) cubeon-cube growth will facilitate a surface with small roughness, often without any distinguishable structural features. Growth in the fast direction (111) often results in triangular spikes. Sometimes, octahedron-shaped crystallites can be observed. We do not observe crystallites with any obvious repeated shape, but we do indeed see a much rougher and spiked surface on the $\mathrm{Al}_{2} \mathrm{O}_{3}$ (001). With this, we believe that it is safe to conclude that epitaxial growth of NFO is indeed achieved on a range of substrates.

\section{SUMMARY AND CONCLUSIONS}

Iron-rich nickel ferrite spinel is grown epitaxially as deposited by atomic layer deposition at a temperature of $250{ }^{\circ} \mathrm{C}$. The films require no postannealing to obtain control of the orientation. Annealing the films at $650^{\circ} \mathrm{C}$ for $15 \mathrm{~min}$ leads to strain relaxation, but no change in crystal orientation. The films with (100)-, (110)-, and (111)-orientation are obtained by careful choice of substrate, taking lattice matches into account. This effectively means that the easy axis of magnetization can be selected to lie in a suitable direction for a specific application. The magnetic hardness of the film will change upon the direction of the easy axis. Control of the easy axis at these low temperatures is very rare and will have a major impact on the possibility to grow multiferroic stacks and on monolithic device integration where a spontaneously magnetized sample is necessary.

We also evaluated the growth dynamics of the deposited films by looking at the growth per cycle as a function of the number of cycles and by studying the surface morphology by AFM. The observed growth is in remarkably good agreement with theoretical epitaxial growth. Understanding the growth dynamics leads to invaluable information regarding nucleation onset and growth of very thin films. These effects have to be taken into account when controlling the thickness and uniformity of deposited layers on different substrates.

We finally confirm that growth inhibition during the initial cycles is mostly related to iron, leading to a slightly nickel-rich phase for the first $10 \mathrm{~nm}$ of the film growth. This composition gradient is, however, small and should not interfere with functional properties.

\section{ACKNOWLEDGMENTS}

The authors would like to acknowledge the beamline staff at ID03@ESRF for fruitful collaboration. They would also like to thank the Department of Geology, University of Oslo for use of the XRF-equipment. This work was partially performed within the RIDSEM-project, financed in full by the Research Council of Norway (Project No. 272253).

${ }^{1}$ Y. P. Sukhorukov, A. V. Telegin, N. G. Bebenin, A. P. Nosov, V. D. Bessonov, and A. A. Buchkevich, Solid State Commun. 263, 27 (2017).

${ }^{2}$ K. J. Kim, H. S. Lee, M. H. Lee, and S. H. Lee, J. Appl. Phys. 91, 9974 (2002).

${ }^{3}$ B. S. Holinsworth, D. Mazumdar, H. Sims, Q.-C. Sun, M. K. Yurtisigi, S. K. Sarker, A. Gupta, W. H. Butler, and J. L. Musfeldt, App. Phys. Lett. 103, 082406 (2013).

${ }^{4}$ M. H. Kryder, Thin Solid Films 216, 174 (1992).

${ }^{5}$ D. Venkateshvaran, M. Althammer, A. Nielsen, S. Geprägs, M. S. Ramachandra Rao, S. T. B. Goennenwein, M. Opel, and R. Gross, Phys. Rev. B 79, 134405 (2009).

${ }^{6}$ J. S. Andrew, J. D. Starr, and M. A. K. Budi, Scr. Mater. 74, 38 (2014).

${ }^{7}$ U. Lüders et al., Adv. Mater. 18, 1733 (2006).

${ }^{8}$ L. Sandlund, M. Fahlander, T. Cedell, A. E. Clark, J. B. Restorff, and M. Wun-Fogle, J. Appl. Phys. 75, 5656 (1994).

${ }^{9}$ G. Sreenivasulu, H. Qu, and G. Srinivasan, Sci. Tech. 30, 1625 (2014).

${ }^{10}$ K. Chand Verma, V. Pratap Singh, M. Ram, J. Shah, and R. K. Kotnala, J. Magn. Magn. Mater. 323, 3271 (2011).

${ }^{11}$ J. Azadmanjiri, S. A. Seyyed Ebrahimi, and H. K. Salehani, Ceram. Int. 33, 1623 (2007).

${ }^{12}$ R. Grigalaitis, M. M. Vijatović Petrović, J. D. Bobić, A. Dzunuzovic, R. Sobiestianskas, A. Brilingas, B. D. Stojanović, and J. Banys, Ceram. Int. 40, 6165 (2014).

${ }^{13}$ Y. Liu, Y. Wu, D. Li, Y. Zhang, J. Zhang, and J. Yang, J. Mater. Sci. Mater. Electron. 24, 1900 (2013).

${ }^{14} \mathrm{~N}$. Ortega, A. Kumar, and R. S. Katiyar, presented at the 17th IEEE International Symposium on the Applications of Ferroelectrics, Santa Fe, NM, 24 February 2008 (IEEE, 2008).

${ }^{15}$ M. T. Johnson, P. G. Kotula, and C. B. Carter, J. Cryst. Growth 206, 299 (1999).

${ }^{16}$ M. Hoppe, S. Döring, M. Gorgoi, S. Cramm, and M. Müller, Phys. Rev. B 91, 054418 (2015).

${ }^{17}$ R. Datta, B. Loukya, N. Li, and A. Gupta, J. Cryst. Growth 345, 44 (2012).

${ }^{18}$ Y. Yang, Q. Tao, G. Srinivasan, and C. G. Takoudis, ECS J. Solid State Sci. 3, 345 (2014).

${ }^{19}$ C. Klewe, M. Meinert, A. Boehnke, K. Kuepper, E. Arenholz, A. Gupta, J.-M. Schmalhorst, T. Kuschel, and G. Reiss, J. Appl. Phys. 115, 123903 (2014).

${ }^{20}$ M. George, A. Mary John, S. S. Nair, P. A. Joy, and M. R. Anantharaman, J. Magn. Magn. Mater. 302, 190 (2006).

${ }^{21}$ Y. T. Chong, E. M. Y. Yau, K. Nielsch, and J. Bachmann, Chem. Mater. 22, 6506 (2010).

${ }^{22}$ S. M. George, Chem. Rev. 110, 111 (2010).

${ }^{23}$ H. H. Sønsteby, H. Fjellvåg, and O. Nilsen, Adv. Mater. Interfaces 4, 1600903 (2017). 
${ }^{24}$ N. Pachauri, B. Khodadadi, A. V. Singh, J. B. Mohammadi, R. L. Martens, P. R. LeClair, C. Mewes, T. Mewes, and A. Gupta, J. Magn. Magn. Mater. 417, 137 (2016).

${ }^{25}$ D. Nečas and P. Klapetek, Cent. Eur. J. Phys. 10, 181 (2012).

${ }^{26}$ A. B. F. Martinson, M. J. DeVries, J. A. Libera, S. T. Christensen, J. T. Hupp, M. J. Pellin, and J. W. Elam, J. Phys. Chem. C 115, 4333 (2011).

${ }^{27}$ M. Utriainen, M. Kröger-Laukkanen, L.-S. Johansson, and L. Niinistö, Appl. Surf. Sci. 157, 151 (2000).

${ }^{28}$ D. M. Hausmann and R. G. Gordon, J. Cryst. Growth 249, 251 (2003).
${ }^{29}$ J. Dendooven, S. Pulinthanathu Sree, K. De Keyser, D. Deduytsche, J. A. Martens, K. F. Ludwig, and C. Detavernier, J. Phys. Chem. C 115, 6605 (2011)

${ }^{30}$ R. K. Grubbs, C. E. Nelson, N. J. Steinmetz, and S. M. George, Thin Solid Films 467, 16 (2004).

${ }^{31}$ M. Ylilammi, Thin Solid Films 279, 124 (1996).

${ }^{32}$ O. Nilsen, O. B. Karlsen, A. Kjekshus, and H. Fjellvåg, Thin Solid Films 515, 4538 (2007).

${ }^{33}$ See supplementary material at https://doi.org/10.1116/1.5082012 for XRD Williamson-Hall plots and calculations for theoretical growth rate. 